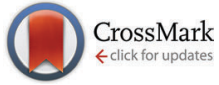

Cite this: Chem. Commun., 2014, 50, 12486

Received 19th June 2014, Accepted 22nd August 2014

DOI: $10.1039 / \mathrm{c} 4 \mathrm{cc} 04683 \mathrm{~h}$

www.rsc.org/chemcomm

\section{Omnidispersible poly(ionic liquid)-functionalized cellulose nanofibrils: surface grafting and polymer membrane reinforcement $\dagger$}

\author{
Konrad Grygiel, ${ }^{a}$ Bernd Wicklein, ${ }^{\mathrm{b}}$ Qiang Zhao, ${ }^{a}$ Michaela Eder, ${ }^{\mathrm{c}}$ \\ Torbjörn Pettersson, ${ }^{d}$ Lennart Bergström, ${ }^{* b}$ Markus Antonietti ${ }^{a}$ and Jiayin Yuan ${ }^{\star a}$
}

We report a facile one-step route to graft poly(ionic liquid)s (PILs) onto cellulose nanofibrils (CNFs). The dispersibility of the PIL-functionalized CNFs in water and various organic solvents could be tuned by the choice of the PIL-binding anion. We demonstrate that such omnidispersible PILCCNF hybrids can be used to reinforce porous poly(ionic liquid) membranes.

Nanocellulose is a renewable nanomaterial with outstanding properties that is attracting rapidly growing scientific and technological interest with applications such as use in composites, barrier films, scaffold materials, multifunctional foams and aerogels. ${ }^{1-3}$ Nanocellulose combines a versatile chemical-modification capacity, low thermal expansion, and excellent mechanical properties with a high surface area. There are three major forms of nanocellulose: cellulose nanofibrils, cellulose nanocrystals, and bacterial cellulose, which mainly differ with respect to dimensions and flexibility. Cellulose nanofibrils (CNFs) are long, slender and flexible rod-like particles with diameters of $\sim 5-60 \mathrm{~nm}$ and lengths up to several $\mu \mathrm{m}$. Due to the high aspect ratio, CNF dispersions are prone to gelation and display pronounced viscoelastic properties even at low concentrations. ${ }^{2}$ CNFs are currently employed (or being introduced) in the food, hygiene, and packaging industry, as additives in cosmetics and tablets, and as scaffold materials in tissue engineering. ${ }^{2,4}$ They were also used to produce mechanically strong films (nanopapers) with tensile strengths up to $250 \mathrm{MPa}^{2}$

\footnotetext{
${ }^{a}$ Department of Colloid Chemistry, Max Planck Institute of Colloids and Interfaces, Am Mühlenberg 1 OT Golm, D-14476 Potsdam, Germany.

E-mail: jiayin.yuan@mpikg.mpg.de

${ }^{b}$ Department of Materials and Environmental Chemistry, Stockholm University, Svante Arrhenius väg 16C, Stockholm, Sweden.

E-mail: lennart.bergstrom@mmk.su.se

${ }^{c}$ Department of Biomaterials, Max Planck Institute of Colloids and Interfaces, Am Mühlenberg 1 OT Golm, D-14476 Potsdam, Germany

${ }^{d}$ Department of Fibre and Polymer Technology, KTH Royal Institute of Technology, Teknikringen 56-58, SE-100 44, Stockholm, Sweden

$\dagger$ Electronic supplementary information (ESI) available. See DOI: 10.1039/ c4cc04683h
}

To achieve good material properties CNFs need to be well dispersed in a suitable medium. Current manufacturing of CNFs typically results in materials with high surface charge, which are dispersible in water but aggregate easily in organic media. This limits the application scope of CNFs in fields where material processing in organic media is unavoidable. To improve dispersibility in organic solvents and/or polymeric matrixes, different strategies such as adsorption or grafting of surfactants, polymers and coupling agents as well as corona treatment have been used. ${ }^{5-11}$ CNFs have also been hydrophobized by acetylation, silylation or "click chemistry". ${ }^{2-14}$ However, many of these routes have limited versatility and some of them, e.g. silylation methods, may damage the fibrillar structure of CNFs. ${ }^{15}$ This highlights the necessity for facile and versatile surface modification methods for the preparation of omnidispersible CNFs that can be easily processed in a wide range of solvents.

Poly(ionic liquid)s (PILs) are strong polyelectrolytes obtained from ionic liquid monomers. ${ }^{16,17}$ PILs retain some features of ILs while showing enhanced mechanical stability and processability due to their polymeric nature. Bearing a high density of IL moieties in the polymeric chain brings new opportunities to combine IL chemistry with polymer research and materials design. ${ }^{18-20}$ For instance, PILs show tunable anion-dependent solubility, which could be related to the binding strength of anions and for particular systems could be presented in the form of Hofmeister series. ${ }^{21-28}$ They also appear to be effective stabilizers for nanomaterials, such as carbon nanotubes, graphene, hydrothermal carbons, and inorganic nanoparticles. ${ }^{23,29-33}$ Especially PIL-stabilized single-walled and multi-walled carbon nanotube dispersions in water are noteworthy due to high achieved concentrations (up to $1.37 \mathrm{wt} \%$ and $17 \mathrm{wt} \%$ of carbon material, respectively), which may be of great importance in further practical applications. ${ }^{34,35}$ Recently, our group has demonstrated the potential of PILs as components of polyelectrolyte membranes, which has attracted attention in both science and industry due to the multitude of possible applications of such systems (e.g., gas separation, controlled release, sensors or catalyst supports). We have developed a straightforward pathway for 

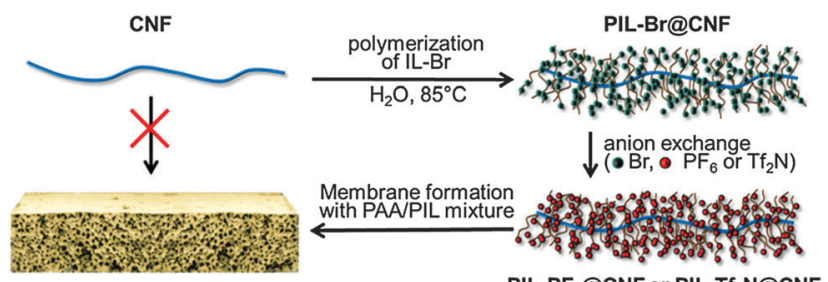

PIL-PF $@ @ C N F$ or PIL-Tf 2 N@CNF

Fig. 1 Synthetic route to a CNF-reinforced porous PIL membrane via one-step surface grafting of a PIL onto CNF (IL-Br: ionic liquid monomer with $\mathrm{Br}^{-}$anion).

preparation of porous membranes by triggering ionic complexation between a PIL and poly(acrylic acid) (PAA). ${ }^{36}$ However, reinforcement of the as-prepared systems is required in order to deal with the problem of their unsatisfactory mechanical properties.

Herein, we report a versatile method to disperse CNFs in water and organic solvents (with different dielectric constants) via one-step grafting of PILs onto CNFs (PIL@CNF). The PIL@CNF hybrids are then exemplarily applied to reinforce the mechanical properties of porous PIL membranes. The overall synthetic scheme is illustrated in Fig. 1.

CNFs were first sonicated in water to obtain a suspension of untangled fibers. PIL chains were grafted onto the negatively charged surfaces of TEMPO (2,2,6,6-tetramethylpiperidine-1oxyl)-oxidized CNFs, by in situ polymerization of an ionic liquid, 1-ethyl-3-vinylimidazolium bromide (IL-Br) in water (at a weight ratio of IL-Br: $\left.\mathrm{CNF}=40: 1, c_{\mathrm{CNF}} \sim 0.05 \mathrm{wt} \%\right)$. A gel-like state was reached during polymerization. After polymerization the hybrids were purified to remove the free polymer and were concentrated (to $0.1 \mathrm{wt} \%$ of $\mathrm{CNF}$ ) by ultrafiltration (pore size: $50 \mathrm{~nm}$ ) using water as an eluent. We confirmed by atomic force microscopy (Fig. S1, ESI $\dagger$ ) that the bundle-free fiber morphology of CNFs is well-preserved after the polymerization and no degradation of the fibers due to their dissolution in reaction media occurred. Moreover, Fourier transform infrared (FTIR) spectroscopy was used in order to confirm the co-existence of PIL-Br and CNFs in the hybrid material (PIL-Br@CNF). The spectra (Fig. 2A) show peaks at $1050 \mathrm{~cm}^{-1}$ (C-O stretching vibration of CNFs), at 1160 and $1550 \mathrm{~cm}^{-1}$ (C-N stretching of imidazolium rings and ring in-plane asymmetric stretching, $\mathrm{CH}_{2}(\mathrm{~N})$ as well as $\mathrm{CH}_{3}(\mathrm{~N}) \mathrm{CN}$ stretching vibrations, respectively),

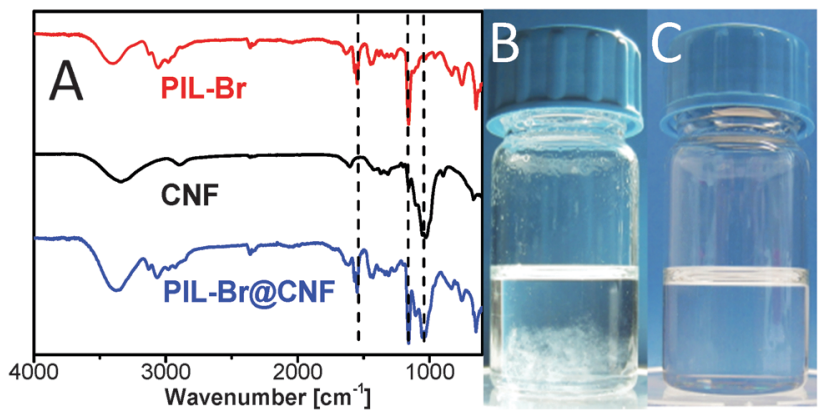

Fig. 2 (A) FTIR spectra of PIL-Br, CNF and the PIL-Br@CNF hybrid; Photos of dispersions of $(B) C N F$ and $(C)$ the $\mathrm{PIL}^{-\mathrm{PF}_{6}} \mathrm{QCNF}$ hybrid in acetone. which verifies the presence of both components in the hybrid product. $^{37}$

The successful grafting of PILs on the surface of CNFs expands the hydrodynamic radius and inverts the surface charge of CNFs. Dynamic light scattering measurement proves that the size of the CNFs increases from 500 to $700 \mathrm{~nm}$ after grafting of PILs. Zeta potential measurements verified that negatively charged CNFs $(-45.4 \pm 4.7 \mathrm{mV})$ became positive $(37.3 \pm 4.2 \mathrm{mV})$ after polymerization. This indicates that a cationic PIL brush layer is created on the CNF surface during the reaction. The CNFs to PIL weight ratio in the hybrid was determined by elemental analysis to be 1 to 2.5 according to the $\mathrm{C} / \mathrm{N}$ weight ratio. The presence of $c a .70 \mathrm{wt} \%$ of PIL in the hybrid confirms the effective growth of PILs onto CNFs via the current one-step grafting method.

Thermogravimetric analysis was conducted to determine the thermal stability of CNFs before and after PIL functionalization. The electrostatic grafting of PIL chains onto the CNF surface did not cause any deterioration in the thermal stability of CNFs (Fig. S2, ESI $\dagger$ ). The temperature needed to create a $10 \mathrm{wt} \%$ mass loss of the CNFs is increased by $20{ }^{\circ} \mathrm{C}$ after grafting of the PIL. Furthermore, the residual mass between 330 and $900{ }^{\circ} \mathrm{C}$ is higher for PIL-Br@CNF than that for pure PIL but lower than that for CNFs, verifying again the presence of both components in the hybrid.

The in situ polymerization-induced stabilization requires no surface pre-functionalization of CNFs. It should be noted that via direct mixing of aqueous solution/dispersion of PILs and CNFs, nondispersible aggregates were formed even upon sonication. This aggregation is caused by electrostatic interaction between these two oppositely charged species. In our in situ polymerization route, we suspect that after addition of several monomeric units to the radical, the short polymer chains adsorb onto CNFs due to electrostatic interactions. The short length of oligomeric PIL chains at this step is the reason why interaction with individual CNF is preferred rather than crosslinking CNFs into an intermolecular network. Due to sorption of short PIL chains, the surface charge of CNFs is first neutralized and then becomes positive with further propagation of the PIL chain polymerization. This induces electrostatic repulsion between particular hybrids as well as between hybrids and free PIL chains in the solution and prevents undesired crosslinking.

The number-average molecular weight $\left(M_{\mathrm{n}}\right)$ of the free PIL chains was determined by gel permeation chromatography to be $35 \mathrm{~kg} \mathrm{~mol}^{-1}$ (using pullulan standards). It was assumed that $M_{\mathrm{n}}$ of both grafted and free PILs obtained during the in situ stabilization of CNFs are comparable, which allows us to roughly calculate grafting density of the polymer chains on the CNF surface (detailed information in ESI $\dagger$ ). The calculated grafting density amounted to 0.15 chain per $\mathrm{nm}^{2}$. Thus, the PIL@CNF hybrid could be considered as a moderate density brush. ${ }^{38}$

One reason for grafting PILs onto CNFs is to control and enable the dispersibility of CNFs in a wide range of organic solvents via the well-known anion-effect of PILs. ${ }^{28,39}$ For example, $\mathrm{Br}^{-}$anions in PIL-Br@CNF were exchanged with hydrophobic $\mathrm{PF}_{6}{ }^{-}$and $\mathrm{TF}_{2} \mathrm{~N}^{-}$anions in order to form PIL-PF$@$ @CNF and 
Table 1 Dispersibility tests of CNF and PIL@CNF hybrids with different anions in various solvents

\begin{tabular}{lllll}
\hline Material & Water & MeOH-EtOH & DMSO & Acetone \\
\hline CNF & + & - & + & - \\
PIL-Br@CNF & + & - & $+/-$ & - \\
PIL-PF 6 @CNF & - & + & + & + \\
PIL-TF 2 N@CNF & - & - & + & -
\end{tabular}

PIL- $\mathrm{TF}_{2} \mathrm{~N} @ \mathrm{CNF}$ hybrids, respectively. The dispersibility tests of the anion-exchanged hybrids were performed and are summarized in Table 1. The PIL-Br@CNF hybrid is dispersible in water but only partially in DMSO. The PIL-TF 2 N@CNF hybrid is hydrophobic and only dispersible in DMSO. Interestingly, the PIL-PF 6 @CNF hybrid is fairly dispersible not only in DMSO but also in acetone and methanol, which are poor solvents for pure, unmodified CNFs (Fig. 2B and C). Hence, without changing the polymer backbone type, the hybrid can be dispersed in a range of solvents, showing that PIL grafting can produce an omnidispersible material.

The tunable dispersibility of the hybrid in organic solvents together with high charge density of this novel material opens up new application possibilities for CNFs, such as for enhancing the mechanical strength of polyelectrolyte-based materials. In this work, we examined the effect of mechanical reinforcement by incorporating PIL@CNF hybrids into porous PIL membranes, prepared via electrostatic complexation triggered by aqueous ammonia. ${ }^{36}$ For the membrane preparation, the PIL-PF 6 @CNF hybrid was added to a mixture of PAA and poly(1-cyanomethyl-3vinylimidazolium $\mathrm{PF}_{6}$ ) in DMSO to form a stable dispersion. This mixture was then cast onto a glass plate, dried and immersed in aqueous ammonia to form a CNF-reinforced porous membrane. Details on the pore formation mechanism can be found in our previous report. ${ }^{36}$ As shown in the scanning electron micrographs (SEMs) (Fig. 3), three-dimensionally connected porous structures were formed in both CNF-free and CNF-containing membranes. The CNF-free membrane and the reinforced membrane display a two-zone morphology (see white lines in Fig. 3A and C) consisting of a top zone with micropores and a bottom bulk zone with pores

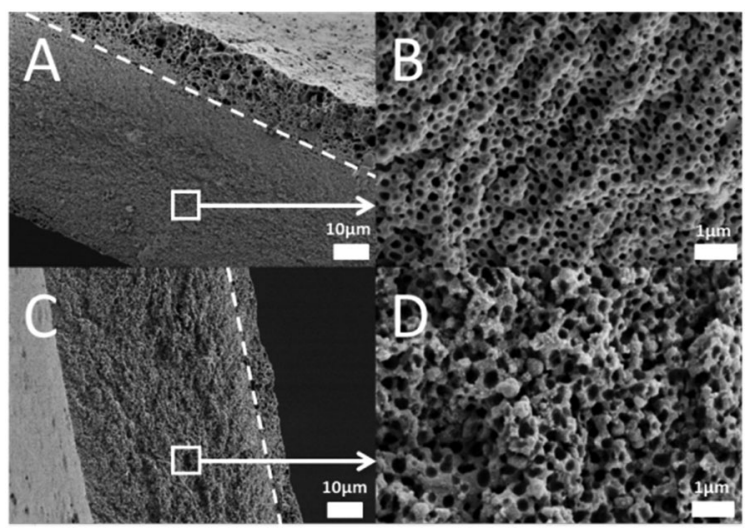

Fig. 3 SEM images of membranes containing: A, B - 0\%; C, D - 5 wt \% of PIL-PF6@CNF. of $150 \pm 50 \mathrm{~nm}$ for the CNF-free (Fig. 3B) and $250 \pm 40 \mathrm{~nm}$ for the reinforced membrane (Fig. 3D).

In order to evaluate the reinforcement of the porous membranes by PIL-PF 6 @CNF hybrids, the mechanical properties of membranes were tested in the wet state. Thin strips of membranes $(14 \times 2 \times 0.1 \mathrm{~mm}, n>5)$ were stressed in tension in a custom-made device. Due to the reinforcement Young's modulus and stress at failure values increased from $\sim 470$ to $\sim 610 \mathrm{MPa}$ and from $\sim 7.8$ to $\sim 10.4 \mathrm{MPa}$, respectively (Fig. S5 and S6, ESI $\dagger$ ). The results show that the incorporated PIL@CNF hybrid, although only $5 \mathrm{wt} \%$, can substantially improve the mechanical performance of the porous PIL membrane. In addition, the reinforced membrane exhibits satisfactory mechanical properties, outrunning stress at failure and Young's modulus values of comparable porous membranes composed of polyethersulfonate, polysulfone, or other polyelectrolyte complexes (Table S1, ESI $\dagger$ ).

In conclusion, we demonstrated an efficient method for facile functionalization of CNFs by PILs via a simple in situ polymerization step. The as-synthesized PIL@CNF hybrids display excellent, dispersibility in water and various organic solvents, which is tunable via simple anion exchange. The omnidispersibility of the PIL-functionalized CNFs enables their application in fields where material processing in organic media is unavoidable. Furthermore, we could show that the addition of PIL@CNF hybrids substantially reinforces porous PIL membranes. The PIL-functionalisation offers a versatile and facile route to create omnidispersible nanoparticles, which opens new avenues to process new composite materials from previously incompatible components.

The authors acknowledge financial support from the Max Planck Society and the Marie Curie Actions of EU's 7th Framework Programme under REA grant agreement no. 289347. The authors thank the HINT-COST action MP1202. BW, TP and LB acknowledge the Wallenberg Wood Science Centre for funding. LB also acknowledges the Humboldt foundation for financial support.

\section{Notes and references}

1 R. J. Moon, A. Martini, J. Nairn, J. Simonsen and J. Youngblood, Chem. Soc. Rev., 2011, 40, 3941-3994.

2 D. Klemm, F. Kramer, S. Moritz, T. Lindström, M. Ankerfors, D. Gray and A. Dorris, Angew. Chem., Int. Ed., 2011, 50, 5438-5466.

3 S. Prescher, F. Polzer, Y. Yang, M. Siebenbürger, M. Ballauff and J. Yuan, J. Am. Chem. Soc., 2013, 136, 12-15.

4 H. Sehaqui, Q. Zhou, O. Ikkala and L. A. Berglund, Biomacromolecules, 2011, 12, 3638-3644.

5 N. Lin, G. Chen, J. Huang, A. Dufresne and P. R. Chang, J. Appl. Polym. Sci., 2009, 113, 3417-3425.

6 H. Lönnberg, K. Larsson, T. Lindström, A. Hult and E. Malmström, ACS Appl. Mater. Interfaces, 2011, 3, 1426-1433.

7 K. Littunen, U. Hippi, L.-S. Johansson, M. Österberg, T. Tammelin, J. Laine and J. Seppälä, Carbohydr. Polym., 2011, 84, 1039-1047.

8 E. Karabulut, T. Pettersson, M. Ankerfors and L. Wågberg, ACS Nano, 2012, 6, 4731-4739.

9 J. M. Felix and P. Gatenholm, J. Appl. Polym. Sci., 1991, 42, 609-620. 10 L. Heux, G. Chauve and C. Bonini, Langmuir, 2000, 16, 8210-8212.

11 C. Aulin, A. Shchukarev, J. Lindqvist, E. Malmström, L. Wågberg and T. Lindström, J. Colloid Interface Sci., 2008, 317, 556-567.

12 D.-Y. Kim, Y. Nishiyama and S. Kuga, Cellulose, 2002, 9, 361-367.

13 G. Siqueira, J. Bras and A. Dufresne, Langmuir, 2009, 26, 402-411.

14 I. Filpponen, E. Kontturi, S. Nummelin, H. Rosilo, E. Kolehmainen, O. Ikkala and J. Laine, Biomacromolecules, 2012, 13, 736-742. 
15 K. Missoum, M. Belgacem and J. Bras, Materials, 2013, 6, 1745-1766. 16 H. Ohno, Macromol. Symp., 2007, 249-250, 551-556.

17 J. Lu, F. Yan and J. Texter, Prog. Polym. Sci., 2009, 34, 431-448.

18 E. B. Anderson and T. E. Long, Polymer, 2010, 51, 2447-2454.

19 H. Chen, J.-H. Choi, D. Salas-de la Cruz, K. I. Winey and Y. A. Elabd, Macromolecules, 2009, 42, 4809-4816.

20 J. Yuan, D. Mecerreyes and M. Antonietti, Prog. Polym. Sci., 2013, 38, 1009-1036.

21 O. Mel'nik, A. Shaplov, E. Lozinskaya, N. Popova, M. Makarov, I. Odinets, K. Lysenko, G. Timofeeva, I. Malyshkina and Y. Vygodskii, Polym. Sci., Ser. B, 2010, 52, 316-326.

22 C. Detrembleur, A. Debuigne, M. Hurtgen, C. Jéröme, J. Pinaud, M. v. Fèvre, P. Coupillaud, J. Vignolle and D. Taton, Macromolecules, 2011, 44, 6397-6404.

23 R. Marcilla, M. Curri, P. Cozzoli, M. Martínez, I. Loinaz, H. Grande, J. Pomposo and D. Mecerreyes, Small, 2006, 2, 507-512.

24 R. Marcilla, J. A. Blazquez, R. Fernandez, H. Grande, J. A. Pomposo and D. Mecerreyes, Macromol. Chem. Phys., 2005, 206, 299-304.

25 R. Marcilla, J. Alberto Blazquez, J. Rodriguez, J. A. Pomposo and D. Mecerreyes, J. Polym. Sci., Part A: Polym. Chem., 2004, 42, 208-212. 26 F. Yan and J. Texter, Chem. Commun., 2006, 2696-2698.

27 D. England, N. Tambe and J. Texter, ACS Macro Lett., 2012, 1, 310-314.
28 J. Texter, Macromol. Rapid Commun., 2012, 33, 1996-2014.

29 D. Mecerreyes, Prog. Polym. Sci., 2011, 36, 1629-1648.

30 D. Batra, S. Seifert, L. M. Varela, A. C. Y. Liu and M. A. Firestone, Adv. Funct. Mater., 2007, 17, 1279-1287.

31 Y. Men, X.-H. Li, M. Antonietti and J. Yuan, Polym. Chem., 2012, 3, 871-873.

32 C. Giordano, W. Yang, A. Lindemann, R. Crombez and J. Texter, Colloids Surf., A, 2011, 374, 84-87.

33 L. Zhao, R. Crombez, F. P. Caballero, M. Antonietti, J. Texter and M.-M. Titirici, Polymer, 2010, 51, 4540-4546.

34 M. Antonietti, Y. Shen, T. Nakanishi, M. Manuelian, R. Campbell, L. Gwee, Y. A. Elabd, N. Tambe, R. Crombez and J. Texter, ACS Appl. Mater. Interfaces, 2010, 2, 649-653.

35 J. Texter, D. Ager, V. A. Vasantha, R. Crombez, D. England, X. Ma, R. Maniglia and N. Tambe, Chem. Lett., 2012, 41, 1377-1379.

36 Q. Zhao, M. Yin, A. P. Zhang, S. Prescher, M. Antonietti and J. Yuan, J. Am. Chem. Soc., 2013, 135, 5549-5552.

37 N. E. Heimer, R. E. Del Sesto, Z. Meng, J. S. Wilkes and W. R. Carper, J. Mol. Liq., 2006, 124, 84-95.

38 L. C. H. Moh, M. D. Losego and P. V. Braun, Langmuir, 2011, 27, 3698-3702.

39 F. Yan and J. Texter, Angew. Chem., Int. Ed., 2007, 119, 2492-2495. 